\title{
Intervenções farmacêuticas em utentes em uso de insulinas análogas sob demanda judicial assistidos no Centro de Especialidades Médico Odontológica, em Belém,
}

\section{Pará}

\author{
Pharmaceutical interventions in patients using insulin analogues under judicial demand assisted at \\ the Centro de Especialidades Médico Odontológica, in Belém, Pará \\ Intervenciones farmacéuticas en pacientes que utilizan análogos de insulina bajo demanda judicial \\ atendidos en el Centro de Especialidades Médico Odontológica, en Belém, Pará
}

Recebido: 27/03/2021 | Revisado: 02/04/2021 | Aceito: 09/04/2021 | Publicado: 19/04/2021

\author{
Yasmin Hanna Couto Brandão \\ ORCID: https://orcid.org/0000-0001-7621-9162 \\ Universidade Federal do Pará, Brasil \\ E-mail: hannabrandao@gmail.com \\ André Lucas de Oliveira Vieira \\ ORCID: https://orcid.org/0000-0002-7571-2286 \\ Universidade Federal do Pará, Brasil \\ E-mail: andreluk47@gmail.com \\ Orenzio Soler \\ ORCID: http://orcid.org/0000-0003-2246-0019 \\ Universidade Federal do Pará, Brasil \\ E-mail: orenziosoler@gmail.com
}

\begin{abstract}
Resumo
Objetivo: Apresentar as intervenções farmacêuticas ofertadas para utentes em uso de insulinas análogas sob judicialização, por meio de serviços farmacêuticos disponibilizados no Centro de Especialidades Médico Odontológica, em Belém, Pará. Método: Trata-se de um estudo de caso, prospectivo, descritivo, analítico de natureza qualitativo e quantitativo, realizado em utentes com Diabetes Mellitus na faixa etária entre 50 e 59 anos, de ambos os sexos e gêneros, em uso de insulinas análogas sob judicialização, por meio da observação participativa, utilizando amostragem de conveniência feito no período entre agosto de 2018 e junho de 2019. Resultados: 27 utentes, sendo 15 do gênero feminino e 12 do gênero masculino, com idade entre 50 e 59 anos integralizaram as 3 Consultas Farmacêuticas. 29,6\% dos utentes apresentavam alguma complicação relacionada ao diabetes; dentre as quais, a prevalência de retinopatia diabética em 50\%. A glicemia capilar casual apresentou uma redução de 215,29 $\pm 80,56$ $\mathrm{mg} / \mathrm{dL}$ para $131 \pm 34,74 \mathrm{mg} / \mathrm{dL}$. A insulina glargina (Lantus®) foi encontrada em $94 \%$ das prescrições. $85,2 \%$ autoreferiram ter adesão ao tratamento. $\mathrm{R} \$ 454,87$ foi o custo médio mensal dos tratamentos dos utentes. $100,0 \%$ dos utentes foram classificados como tendo boa qualidade de vida. Conclusão: Infere-se que as intervenções farmacêuticas demonstraram ser efetivas quanto ao cuidado farmacêutico e a qualidade de vida do utente. Propõe-se, reflexionar, sobre a importância não só da acessibilidade as insulinas análogas via judicial; mas, imprescindivelmente da qualidade do acesso aos serviços de cuidados ofertados.
\end{abstract}

Palavras-chave: Serviço farmacêutico; Intervenção farmacêutica; Judicialização; Insulinas análogas; Diabetes mellitus.

\begin{abstract}
Objective: To present the pharmaceutical interventions offered to users using insulin analogues under judicial review, through pharmaceutical services available at the Centro de Especialidades Médico Odontológica, in Belém, Pará. Method: This is a prospective, descriptive, analytical study of a qualitative and quantitative nature, carried out in patients with Diabetes Mellitus in the age group between 50 and 59 years old, of both sexes and genders, using analogous insulins judicialization, through participatory observation, using convenience sampling carried out between August 2018 and June 2019. Results: 27 users, 15 female and 12 males, aged between 50 and 59 years, completed the 3 Pharmaceutical Consultations. $29.6 \%$ of users had some complication related to diabetes; among which, the prevalence of diabetic retinopathy at $50 \%$. Casual capillary blood glucose decreased from $215.29 \pm 80.56 \mathrm{mg} / \mathrm{dL}$ to $131 \pm 34.74 \mathrm{mg} / \mathrm{dL}$. Insulin glargine (Lantus ${ }^{\circledR}$ ) was found in $94 \%$ of prescriptions. 85.2\% self-reported having adhered to treatment. R \$ 454.87 was the average monthly cost of treatment for users. $100.0 \%$ of users were classified as having a good quality of life. Conclusion: It is inferred that pharmaceutical interventions have been shown to be effective about pharmaceutical care and the quality of life of the user. It is proposed to reflect on the importance not
\end{abstract}


only of accessibility to insulin analogues via the courts; but, fundamentally, the quality of access to the care services offered.

Keywords: Pharmaceutical service; Pharmaceutical intervention; Judicialization; Analogous insulins; Diabetes mellitus.

\section{Resumen}

Objetivo: Presentar las intervenciones farmacéuticas que se ofrecen a los usuarios que utilizan análogos de insulina en revisión judicial, a través de los servicios farmacéuticos disponibles en el Centro de Especialidades Médico Odontológica, en Belém, Pará. Método: Estudio de caso, prospectivo, descriptivo, cualitativo y analítico cuantitativo. estudio, realizado en usuarios con Diabetes Mellitus en el grupo de edad entre 50 y 59 años, de ambos sexos y géneros, utilizando insulinas análogas bajo judicialización, mediante observación participativa, utilizando muestreo de conveniencia realizado en el período comprendido entre agosto de 2018 y junio de 2019. Resultados : 27 usuarios, 15 de los cuales eran mujeres y 12 eran hombres, con edades entre 50 y 59 años, completaron las 3 Consultas Farmacéuticas. El 29,6\% de los usuarios tuvo alguna complicación relacionada con la diabetes; entre los cuales, la prevalencia de retinopatía diabética al 50\%. La glucosa en sangre capilar casual disminuyó de 215,29 $\pm 80,56 \mathrm{mg} / \mathrm{dL}$ a $131 \pm 34,74 \mathrm{mg} / \mathrm{dL}$. Se encontró insulina glargina (Lantus®) en el 94\% de las recetas. El 85,2\% informó haber cumplido el tratamiento. $\mathrm{R} \$ 454,87$ fue el costo promedio mensual de tratamiento para los usuarios. El 100,0\% de los usuarios fueron clasificados como de buena calidad de vida. Conclusión: Se infiere que las intervenciones farmacéuticas han demostrado ser efectivas en cuanto a la atención farmacéutica y la calidad de vida del usuario. Se propone reflexionar sobre la importancia no solo de la accesibilidad a los análogos de insulina a través de los tribunales; pero, fundamentalmente, la calidad del acceso a los servicios de atención ofrecidos.

Palabras clave: Servicio farmacéutico; Intervención farmacéutica; Judicialización; Insulinas análogas; Diabetes mellitus.

\section{Introdução}

Uma das tecnologias de maior custo-efetividade empregado na área da saúde é o medicamento quando utilizado de forma racional. Ao contrário, nos casos de uso de forma inadequada, este se torna um significativo problema de saúde pública1. Para que se tenha uma utilização efetiva e segura dos medicamentos é necessário que eles estejam no foco da atuação dos diferentes profissionais de saúde, de forma a propiciar melhores resultados advindos com a utilização da farmacoterapia e evitar as consequências relacionadas ao seu uso inadequado (Brasil, 2020).

Sabe-se, que as ações da Assistência Farmacêutica restritas à provisão do acesso aos medicamentos, não dão conta de responder às reais necessidades da atenção integral à saúde e minimizar os riscos causados pelo uso inadequado destes. É imprescindível que as pessoas sejam orientadas sobre a utilização apropriada dos medicamentos e a importância do tratamento terapêutico farmacológico para o controle das doenças (Brasil, 2020).

É imprescindível que os serviços farmacêuticos estejam organizados, estruturados e integrados aos demais serviços de saúde, tendo como foco garantir a disponibilidade de medicamentos, assim como a qualidade e conservação; ou seja, a gestão técnica da assistência farmacêutica e a gestão clínica do medicamento (Soler, et al., 2018). Outrossim, no contexto dos serviços farmacêuticos é importante prestar serviços assistenciais, com foco na efetividade e na segurança da terapêutica: avaliar, obter e difundir informações sobre medicamentos e sobre saúde na perspectiva da educação em saúde e da educação permanente das equipes (Brasil, 2020).

Brandão et al. (2021) no estudo sobre o cumprimento do protocolo clínico para atendimento de utentes de análogos de insulina em portadores de Diabetes Mellitus via judicial, avaliaram o cumprimento dos critérios estabelecidos para o atendimento via judicial de análogos de insulinas (lispro [Humalog ${ }^{\circledR}$ ], asparte [NovoRapid®], glulisina [Lispro®], glargina [Lantus $\left.{ }^{\circledR}\right]$, detemir [Levemir $\left.{ }^{\circledR}\right]$, degludeca [Tresiba $\left.{ }^{\circledR}\right]$ ), disponibilizados no Centro de Especialidades Médico Odontológica (CEMO), em Belém, Pará; evidenciado que os critérios estabelecidos são parcialmente cumpridos. 
Evidências inconclusas quanto ao uso de insulinas análogas

As insulinas análogas têm sido alvo de controvérsias relativas à sua superioridade terapêutica em relação às insulinas regular e NPH. Ainda há escassez de evidências científicas de qualidade, que possam comprovar a superioridade terapêutica das análogas em relação às humanas. Não obstante, há solicitações da Sociedade Brasileira de Diabetes (SBD) à Comissão Nacional de Incorporação de Tecnologias (Conitec) quanto à incorporação desses medicamentos no âmbito do Sistema Único de Saúde (SUS). Até 2020 a Relação Nacional de Medicamentos Essenciais (Rename) incluía apenas a insulina humana NPH (de ação intermediária) e a insulina humana regular (de ação rápida), mas não incluía as insulinas análogas, sejam as de ação prolongada (Glargina, Degludeca e Detemir), sejam as de ação ultrarrápida (Asparte, Lispro e Glulisina), introduzidas a partir daquele ano. As insulinas análogas já estavam disponíveis no mercado brasileiro e eram objetos frequentes de ações judiciais no Brasil (Brasil, 2014a; 2014b; Ramos et al., 2017).

A Comissão Nacional de Incorporação de Tecnologias (Conitec) no Sistema Único de Saúde (SUS) recomendou a incorporação das insulinas de ação rápida pelo SUS para tratamento do Diabetes Mellitus Tipo 1 somente em 2017 (BRASIL, 2016). Em 2019 as insulinas de ação prolongada também receberam parecer favorável à incorporação, condicionada ao custo do tratamento ser igual ou inferior ao da insulina NPH, mesmo sem novas evidências científicas de superioridade terapêutica (BRASIL, 2019a).

Ainda em 2019 a Comissão Nacional de Incorporação de Tecnologias (Conitec) torna publica a decisão de não incorporar as insulinas análogas para o tratamento de Diabetes Mellitus Tipo 2 no âmbito do SUS, por meio da Portaria GM/MS nº 11, de 25 de fevereiro de 2019 (Brasil, 2019b), onde após apreciação das contribuições encaminhadas por consulta pública, foram discutidas questões sobre o discreto benefício associado ao uso das insulinas análogas de ação prolongada, as incertezas quanto a redução no risco de crises de hipoglicemia e a importância de uma abordagem terapêutica que incluam medidas de autocuidado, educação em saúde e mudanças de habito de vida.

As revisões da Colaboração Cochrane não encontraram resultados que indiquem que as insulinas análogas apresentam vantagens terapêuticas relevantes sobre as humanas. Demonstram, ainda, que o debate científico sobre o uso das insulinas análogas em relação às humanas é controverso (Fullerton et al, 2016). Mesmo com a falta de evidências sobre vantagens terapêuticas das insulinas análogas em relação às humanas e a despeito do fato da maioria delas não estarem incluídas na Relação Nacional de Medicamentos Essenciais (Rename), os médicos continuam prescrevendo aos utentes e estes têm recorrido ao Poder Judiciário para que o Estado forneça esses medicamentos (Brasil, 2016).

Em Belém, Pará, foi aprovado o Processo n 0006454-87.2008.4.01.3900, de 05 de abril de 2016, do Poder Judiciário do Tribunal Regional Federal da Primeira Região / Seção Judiciária do Estado do Pará, (Pará, 2016), que trata de uma ação civil pública com pedido de tutela antecipada para que o município forneça análogos de insulina e antidiabéticos orais/injetáveis e de todos os insumos necessários à sua aplicação (seringas com agulha acoplada para aplicação de insulina, tiras reagentes de medida de glicemia capilar e lancetes para punção digital), desde que que comprovem a real necessidade do uso desses medicamentos.

Para o cumprimento da ação civil pública ficou estabelecido que o atendimento dos utentes com Diabetes mellitus seria no Centro de Especialidades Médico Odontológica (CEMO), onde hoje há dispensação de 6 tipos de insulinas análogas, sendo 3 de ação ultrarrápida; lispro (Humalog $®$ ), asparte (NovoRapid®), glulisina (Lispro®) e, os análogos de insulina de ação prolongada; glargina (Lantus () , detemir (Levemir®) e degludeca (Tresiba $\left.{ }^{\circledR}\right)$. O atendimento dos utentes deveria cumprir o "Protocolo clínico para atendimento de usuários de análogos de insulina e antidiabéticos orais/ injetáveis para utentes portadores de Diabetes Mellitus da rede pública de saúde do município de Belém” (Belém, 2015; Brandão et al., 2021). 
Neste contexto, o objetivo deste artigo é apresentar as intervenções farmacêuticas ofertadas para utentes em uso de insulinas análogas sob judicialização, por meio de serviços farmacêuticos disponibilizados no Centro de Especialidades Médico Odontológica (CEMO), em Belém, Pará.

\section{Material e Métodos}

\section{Tipo e local do estudo}

Trata-se de um estudo de caso, prospectivo, descritivo, analítico de natureza qualitativo e quantitativo, por meio da observação participativa, utilizando-se de amostragem de conveniência (Freitas, Jabbour, 2011; Minayo, 2012; Thiollent, 2011; Yin, 2015), tendo como recorte temporal o período entre agosto de 2018 e junho de 2019.

\section{Universo do estudo}

Este estudo foi conduzido no Centro de Especialidades Médicas e Odontológicas (CEMO) da Secretaria Municipal de Saúde (SESMA) da Prefeitura Municipal de Belém (PMB), onde em 2018 havia uma população de 2931 utentes sob demanda judicial cadastrados para o recebimento de insulinas análogas. Trata-se de uma Unidade de Especializada de Saúde que realiza atendimento especializado em áreas médicas e odontológicas, além de dispensar análogos de insulinas. A equipe multiprofissional do CEMO conta 09 Neurologistas, 01 Cardiologista, 03 Dermatologistas, 04 Oftalmologistas, 04 Farmacêuticos, 01 Fonoaudióloga, 03 Assistentes Sociais, 01 Enfermeiro, 10 Técnicas de Enfermagem, 15 Técnicas de Saúde Bucal e 30 Odontólogos.

\section{Critérios de inclusão}

Utentes com Diabetes Mellitus na faixa etária entre 50 e 59 anos, de ambos os sexos e gêneros, em uso de insulinas

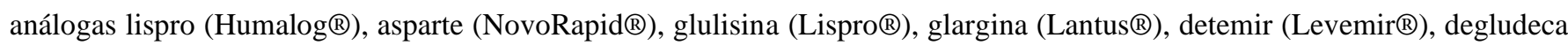
(Tresiba $\left.{ }^{\circledR}\right)$, sob demanda judicial, atendidos no Centro de Especialidades Médicas e Odontológicas (CEMO), os quais assinaram o Termo de Consentimento Livre Esclarecido (TCLE) e que cumpriram todas as etapas de Intervenção Farmacêutica ao longo de seis meses de acompanhamento.

\section{Amostra, coleta de dados e informações}

Trabalhou-se com 27 utentes que cumpriram os critérios de inclusão. Para obter os dados e informações se utilizou de prontuários e de entrevistas. Para obtenção de dados sociodemográficos utilizou-se da ferramenta PROGRESS (O’Neill et al., 2013). Para as intervenções farmacêuticas relacionadas aos desfechos clínicos, epidemiológicos, de acesso (output), humanísticos e econômicos, utilizou-se de protocolos para o cuidado farmacêutico adaptados de modelos oficiais (Brasil, 2014; 2020; CFF, 2016) exemplificado na Figura 1. Para o estudo de potenciais interações de medicamento-medicamento, medicamento-alimento e duplicidade terapêutica, buscou-se bases de dados científicas no Portal Saúde Baseada em Evidências da Universidade Federal do Rio Grande do Norte (https://psbe.ufrn.br/2 e da Universidade Federal do Rio Grande do Sul (http://www.ufrgs.br/bibicbs/pesquisa/portal-saude-baseada-em-evidencias), utilizando-se a classificação de Alta, Média e Baixa Gravidade. Para calcular os custos médios mensais dos tratamentos dos utentes, utilizou-se dos valores disponibilizados pelo Banco de Preços do Ministério da Saúde (http://bps.saude.gov.br/login.jsf). 
Figura 1 - Fluxograma de atendimento para o cuidado farmacêutico de utentes diabéticos em uso de insulinas análogas.

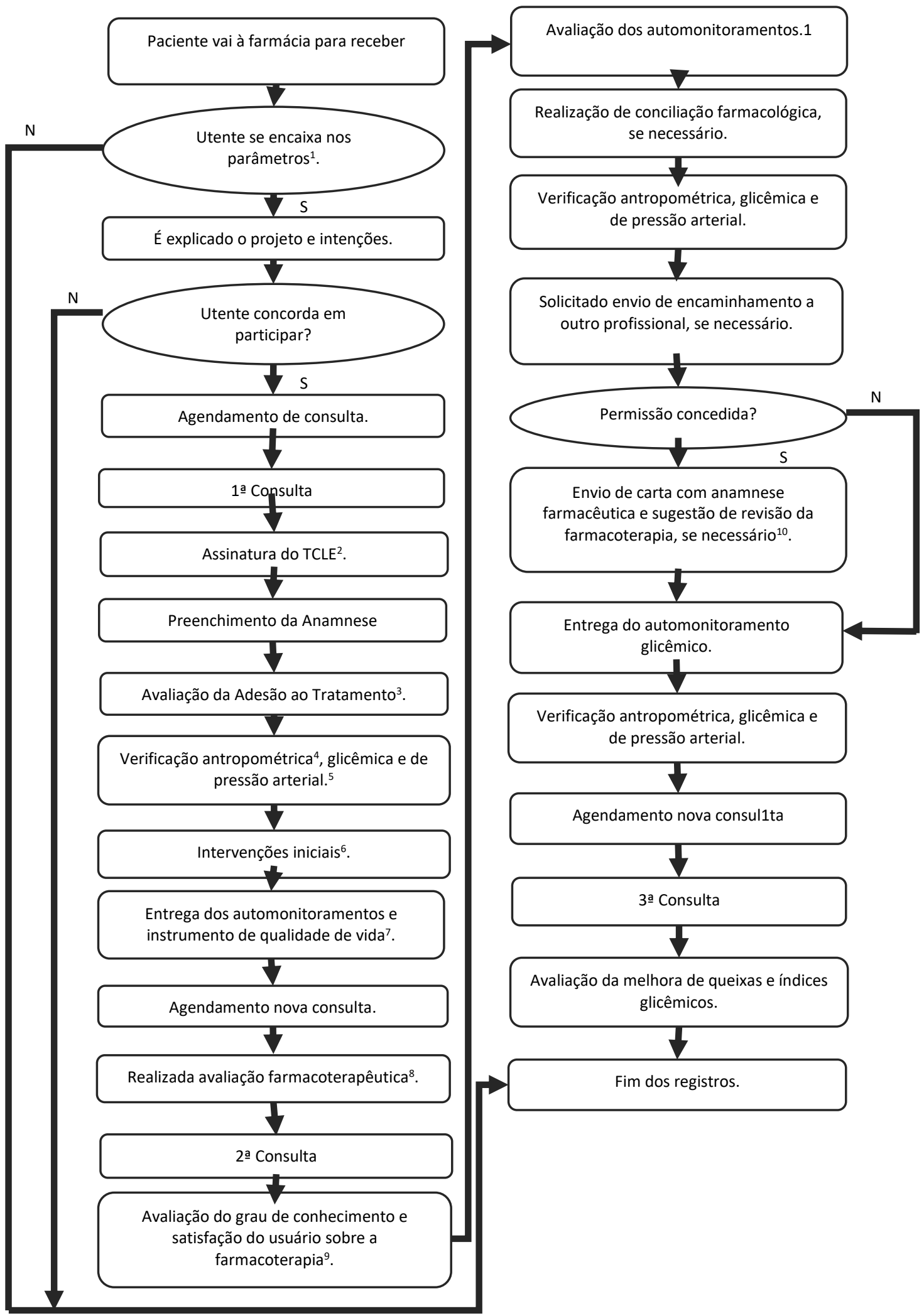

Legenda: ${ }^{1}$ Utente cadastrado na SESMA/PMB e Cemo para recebimento de insulina análoga e idade entre 50 e 59 anos. ${ }^{2}$ Termo de Consentimento Livre e Esclarecido. ${ }^{3}$ Escala Likert. ${ }^{4}$ Verificação de peso e altura. ${ }^{5}$ Avaliações realizadas mediante a presença de um farmacêutico habilitado. ${ }^{6}$ Intervenções referentes a educação em saúde do utente. ${ }^{7}$ Formulário de Grau de Conhecimento do Usuário sobre os Medicamentos Prescritos. ${ }^{8}$ Revisada e assinada por um farmacêutico habilitado. ${ }^{9}$ Instrumento de avaliação de qualidade de vida: WHOQOL. 
Para avaliar a qualidade de vida foi aplicado o instrumento WHOQOL (Fleck et al., 2000), onde o resultado é apresentado em quatro domínios de avaliação: Domínio Físico, Domínio Psicológico, Relações Sociais e Meio ambiente, dispostos em 24 quesitos.

\section{Análise dos dados e apresentação dos resultados}

Os dados foram tratados com estatística descritiva e os resultados estão apresentados ou em frequência absoluta e relativa ou na forma dicotômica de "sim" e "não" ou por meio de síntese narrativa. Apresenta-se os resultados quanto ao perfil de utilização de medicamentos, as intervenções farmacêuticas por meio do cuidado farmacêutico e os desfechos de acesso, epidemiológicos, clínicos, humanísticos e econômicos.

\section{Aspectos éticos}

Esta investigação foi submetida à Plataforma Brasil de acordo com os marcos regulatórios que regulamentam as diretrizes e normas de pesquisas envolvendo seres humanos (Brasil, 2012; 2016), recebendo o CAAE $\mathrm{n}^{\circ}$ 70889717.9.0000.0018 e Parecer Consubstanciado nº 2.179.584, em 20 de julho de 2017.

\section{Resultados e Discussão}

Registra-se que em agosto de 2018 havia 2931 utentes cadastrados no CEMO para recebimento de insulinas análogas, divididos entre os que recebem ativamente, os que pararam de receber, por motivos pessoais e os recém cadastrados, que ainda não havia. Do total de utentes cadastrados, avaliou-se 1149 processos quanto à origem, tipo de Diabetes Mellitus, apresentação de documentos; tais como RG, CPF e cartão SUS, laudo médico de até 6 meses, exames laboratoriais atualizados e indicação de insulina análogas a serem utilizadas. Foram entrevistados 34 utentes, sendo 61,8\% ( $\mathrm{n}=21$ ) do gênero feminino e 38,2\% (n = 13) do gênero masculino, com idade entre 50 e 59 anos, com média de idade \pm desvio padrão de $55,73 \pm 3,00$, sendo $56,04 \pm$ 2,90 anos para mulheres e 55,23 $\pm 3,21$ para homens. Dos 34 utentes que iniciaram o estudo, 27 (79,41\%) utentes integralizaram as 3 Consultas Farmacêuticas; sendo os resultados apresentados a partir desta amostra.

\section{Dados Sociodemográficos}

Quanto ao perfil sociodemográfico dos utentes que completaram o acompanhamento $(n=27 ; 100,0 \%)$, tem-se os seguintes achados (Tabela 1). 
Tabela 1 - Dados sociodemográfico dos utentes.

\begin{tabular}{|c|c|c|}
\hline PROGRESS $*$ & $\mathrm{FA}(\mathrm{n})$ & FR $(\%)$ \\
\hline \multicolumn{3}{|l|}{ Sexo Biológico } \\
\hline Feminino & 15 & $55,6 \%$ \\
\hline Masculino & 12 & $44,4 \%$ \\
\hline \multicolumn{3}{|l|}{ Orientação Sexual } \\
\hline Heterossexual & 27 & $100 \%$ \\
\hline Lésbicas/Bissexuais/Travestis/Transexuais/Transgêneros & 0 & $0 \%$ \\
\hline \multicolumn{3}{|l|}{ Raça/Etnia/Cultura/Idioma } \\
\hline Branca & 6 & $22,2 \%$ \\
\hline Preta & 4 & $14,8 \%$ \\
\hline Parda & 14 & $51,9 \%$ \\
\hline Amarela & 2 & $7,4 \%$ \\
\hline Indígena & 1 & $3,7 \%$ \\
\hline \multicolumn{3}{|l|}{ Formação Educacional } \\
\hline Analfabeto & 1 & $3,8 \%$ \\
\hline Ensino fundamental incompleto & 4 & $14,8 \%$ \\
\hline Ensino fundamental completo & 4 & $14,8 \%$ \\
\hline Ensino médio completo & 9 & $33,3 \%$ \\
\hline Ensino superior completo & 9 & $33,3 \%$ \\
\hline \multicolumn{3}{|l|}{ Ocupação } \\
\hline Aposentado & 8 & $39,6 \%$ \\
\hline Desempregado & 10 & $37 \%$ \\
\hline Profissionais científicos e intelectuais & 5 & $18,5 \%$ \\
\hline Trabalhadores não qualificados & 3 & $11,1 \%$ \\
\hline Outros & 1 & $3,8 \%$ \\
\hline \multicolumn{3}{|l|}{ Renda } \\
\hline Abaixo de 1 salário-mínimo & 5 & $18,5 \%$ \\
\hline Entre 1 e 2 salários-mínimos & 12 & $44,4 \%$ \\
\hline Entre 3 e 4 salários-mínimos & 4 & $14,8 \%$ \\
\hline Entre 5 e 6 salários-mínimos & 4 & $14,8 \%$ \\
\hline Acima de 7 salários-mínimos & 2 & $5,5 \%$ \\
\hline \multicolumn{3}{|l|}{ Local de Residência } \\
\hline Urbano & 27 & $100 \%$ \\
\hline Rural & 0 & $0 \%$ \\
\hline \multicolumn{3}{|l|}{ Habitantes por domicílio } \\
\hline Mora sozinho & 4 & $14,8 \%$ \\
\hline 2 habitantes & 13 & $48,1 \%$ \\
\hline Entre 3 e 5 habitantes & 10 & $37 \%$ \\
\hline \multicolumn{3}{|l|}{ Religião } \\
\hline Catolicismo & 24 & $88,9 \%$ \\
\hline Espiritismo & 1 & $3,7 \%$ \\
\hline Protestantismo & 1 & $3,7 \%$ \\
\hline Agnóstico & 1 & $3,7 \%$ \\
\hline \multicolumn{3}{|l|}{ Entidades e/ou Organismos que se relaciona } \\
\hline Família & 29 & $96,3 \%$ \\
\hline Grupo de amigos & 21 & $77,8 \%$ \\
\hline Academia & 3 & $11,1 \%$ \\
\hline Igreja & 2 & $7,4 \%$ \\
\hline Redes Sociais & 2 & $7,4 \%$ \\
\hline
\end{tabular}

Nota: $\mathrm{P}=$ Local de residência; $\mathrm{R}$ = Raça/etnia/cultura/idioma; $\mathrm{O}=$ Ocupação; $\mathrm{G}=$ Sexo/gênero; $\mathrm{R}=$ Religião; $\mathrm{E}=$ Educação; $\mathrm{S}=$ Estado socioeconômico; $\mathrm{S}=$ Capital social.

Fonte: Perfil de prescrição e de utilização de medicamentos em utentes com diabetes mellitus atendidos via processos judiciais no Centro de Especialidades Médicas e Odontológicas (CEMO) do Município de Belém, Pará.

Observa-se que há um equilíbrio entre homens $(44,4 \%)$ e mulheres $(55,6 \%)$. Quanto a raça/etnia foi encontrado uma prevalência de autodeclarados pardos (51,9\%), seguido de brancos (22,2\%). Quanto ao local de residência, $100 \%$ dos 
entrevistados são residentes em áreas urbanas. Segundo Arruda, Maia \& Alves (2017), há diferenças significativas nas necessidades de cuidados em saúde de acordo com as áreas urbana e rural, demonstrando um percentual extremamente baixo de pessoas nas áreas rurais com facilidade de acesso aos serviços.

Quanto ao nível de escolaridade o número de pessoas com ensino médio completo $(33,3 \%)$ é igual ao número de pessoas com nível superior completo $(33,3 \%)$, os quais junto somam $66,6 \%$, demonstrando um predomínio da população com maior grau de escolaridade; ou seja, melhor nível de esclarecimento. Quanto à ocupação, 30\% relataram ser aposentado e 37\% não exercer nenhuma função formal, dado que repercute diretamente na renda familiar; sendo que $63 \%$ relataram ter uma renda familiar abaixo de 2 salários-mínimos. Vieira (2017), relata que em seu estudo $40 \%$ eram de aposentado e 5\% de desempregado e que $90 \%$ apresentavam uma renda média mensal abaixo de 3 salários-mínimos.

Quanto a religião $88,9 \%$ relataram ser católicos; sendo que 7,4\% frequentam igrejas. A religião e a espiritualidade se apresentam como importante instrumento aos utentes, uma vez que a utilizam como forma de enfrentamento quando se deparam com o diagnóstico e com a terapêutica da doença. A proximidade com o transcendental por meio da oração, contribui para que o utente tenha forças e ânimo para encarar as adversidades impostas pela patologia (Caires, 2016). Quanto ao capital social, $96,3 \%$ se relacionam com a família, 77,8\% com grupos de amigos, 11,15 academia e 7,4\% redes sociais.

\section{Hábitos e estilo de vida}

A mudança de estilo de vida para um utente diabético é imprescindível; uma vez que só o tratamento farmacológico não é suficiente para melhorar seu quadro de saúde. Dentre esses fatores, o consumo de drogas lícitas ou ilícitas são destaque para serem avaliados a fim de assegurar uma boa qualidade de vida, quando esses hábitos são abandonados (Tabela 2). 
Tabela 2 - Hábitos, estilo de vida e uso de plantas medicinais.

\begin{tabular}{|c|c|c|}
\hline PARÂMETROS & FA (n) & FR (\%) \\
\hline \multicolumn{3}{|c|}{ Tabagismo } \\
\hline Não fuma & 25 & $92,6 \%$ \\
\hline Parou de fumar há mais de 1 ano & 2 & $7,4 \%$ \\
\hline \multicolumn{3}{|c|}{ Bebida alcoólica } \\
\hline Beber abaixo de 2 vezes por semana & 9 & $33,3 \%$ \\
\hline Não bebe & 16 & $59,3 \%$ \\
\hline Parou de beber há mais de 1 ano & 2 & $7,4 \%$ \\
\hline Outras drogas & 1 & 3,7 \\
\hline \multicolumn{3}{|c|}{ Pratica exercício físico } \\
\hline Sim & 13 & $48,1 \%$ \\
\hline Não & 14 & $51,9 \%$ \\
\hline \multicolumn{3}{|c|}{ Frequência dos exercícios físicos } \\
\hline $1-2$ vezes por semana & 3 & $23 \%$ \\
\hline $3-5$ vezes por semana & 9 & $69,2 \%$ \\
\hline Acima de 5 vezes por semana & 1 & $7,8 \%$ \\
\hline \multicolumn{3}{|c|}{ Índice de Massa Corporal } \\
\hline \multicolumn{3}{|l|}{ Consulta inicial (IMC) } \\
\hline Abaixo do peso & 2 & $7,4 \%$ \\
\hline Peso adequado & 8 & $29,6 \%$ \\
\hline Sobrepeso & 7 & $25,9 \%$ \\
\hline Obesidade & 10 & $37,1 \%$ \\
\hline \multicolumn{3}{|l|}{ Consulta final (IMC) } \\
\hline Abaixo do peso & 1 & $3,7 \%$ \\
\hline Peso adequado & 10 & $37,1 \%$ \\
\hline Sobrepeso & 7 & $25,9 \%$ \\
\hline Obesidade & 9 & $33,3 \%$ \\
\hline \multicolumn{3}{|c|}{ Uso de plantas medicinais } \\
\hline Não utiliza & 20 & $74,1 \%$ \\
\hline Utiliza & 7 & $25,9 \%$ \\
\hline \multicolumn{3}{|c|}{ Chás utilizados } \\
\hline Chá de Pata de Vaca (Bauhinia forficata) & 3 & $42,9 \%$ \\
\hline Chá de Salsinha (Petroselinum crispum) & 1 & $14,3 \%$ \\
\hline Chá Erva Doce (Pimpinella anisum) & 1 & $14,3 \%$ \\
\hline Outros & 2 & $28,5 \%$ \\
\hline
\end{tabular}

Nota: Os parâmetros indicados pelo Ministério da Saúde para avaliação do estado nutricional de pessoas entre 20 e 59 anos são o Índice de Massa Corporal (IMC) e o perímetro da cintura ou circunferência da cintura. O resultado do cálculo do IMC é analisado de acordo com a classificação definida pela Organização Mundial de Saúde (OMS), válida somente para pessoas adultas: Baixo peso: < 18,5; Peso adequado: $\geq 18,5$ e $<25$, Sobrepeso: $\geq 25$ e $<30$; Obesidade: $\geq 30$.

Fonte: Perfil de prescrição e de utilização de medicamentos em utentes com diabetes mellitus atendidos via processos judiciais no Centro de Especialidades Médicas e Odontológicas (CEMO) do município de Belém, Pará.

3,7\% dos utentes relataram usar algum tipo de droga ilícita; mas se abstiveram em informar a frequência e tipo de uso. Quanto ao consumo de alcoólicos, 33,3\% relataram um consumo inferior à de 2 vezes por semana; ou seja, em "situações ditas especiais" (Tabela 2). Segundo a Sociedade Brasileira de Diabetes (2019), o álcool afeta a alimentação e a glicemia, prejudicando o controle do Diabetes Mellitus, interferindo na ação da insulina, dos secretagogos e do glucagon; aumentando, assim, o risco de hipoglicemia em indivíduos que fazem uso dessas substâncias. Ele pode, também, reduzir os níveis glicêmicos e a consciência, sendo que o álcool e a hipoglicemia têm efeitos adversos independentes, mas aditivos, sobre a função cognitiva.

$100 \%$ dos utentes relataram não ter o hábito do fumo e/ou já o ter sanado há mais de 1 ano. Quanto a prática de exercício físico, 51,8\% relataram fazer alguma atividade e, destes, 64\% praticam entre 3 e 5 vezes por semana atividades físicas (Tabela 2). Caires (2016), menciona que a atividade física é eficaz no controle do Diabetes Mellitus, pois contribui para a diminuição de fatores de risco relacionados ao sistema cardiovascular, e também na redução dos índices glicêmicos. Sabe-se 
que o exercício físico auxilia na perda de peso, diminuição do estresse e melhoria do bem-estar, que influência diretamente no tratamento.

Quanto a obesidade, observou-se após as intervenções farmacêuticas, uma redução do percentual de obesos em 33\% dos utentes; ou seja, o percentual de pessoas com peso adequado passou de 30\% para 37\% (Tabela 2). Na população diabética, o uso intensivo de terapia insulínica e a supervalorização das metas glicêmicas têm resultado em aumento das taxas de hipoglicemia grave e contribuído para a predisposição ao ganho de peso (Masmiquel et al., 2016).

Quando o utente foi questionado quanto ao uso de terapias complementares, houve relatos do uso de chás como adjuvante no seu tratamento, $26 \%$ dos utentes relataram fazer uso de chás medicinais com objetivo de auxiliar no tratamento do diabetes (Tabela 2), estando orientado quanto ao seu uso correto. Dos utentes que relataram fazer uso de chá, $43 \%$ citaram o "chá de pata de vaca" (Bauhinia forficata), o que condiz com o descrito na literatura, onde Vieira (2017) teve como resultados que 38,5\% relatavam fazer o uso do mesmo chá. Não há evidências sobre potenciais efeitos adversos e/ou interações em decorrência do uso concomitante de plantas medicinais e os medicamentos convencionais para o tratamento do Diabetes Mellitus. Existem estudos etnobotânicos que descrevem a existência de cerca de 800 plantas que apresentam potenciais propriedades anti-hiperglicemiantes (Patel, Laloo \& Hemalatha, ${ }^{2012)}$. Outrossim, deve-se ter uma atenção redobrada quanto ao uso concomitante de plantas medicinais e medicamentos hipoglicemiantes.

Importante, registrar, que 100,0\% dos utentes passaram por grupos operativos-educativos sobre a importância de hábitos e estilos de vida saudáveis. A mudança de estilo de vida para um utente diabético é imprescindível, uma vez que só o tratamento farmacológico não é suficiente para melhorar sua qualidade de vida. A prática de exercícios físicos também se mostram imprescindíveis para quem os pode realizar, contribuindo para uma vida mais saudável (Tabela 2).

\section{Dados e informações de diagnóstico e laboratoriais}

Quanto ao tipo de Diabetes Mellitus, 63\% apresentam Diabetes Mellitus Tipo 2, o que condiz com a literatura; visto que é o tipo mais encontrado, segundo a Sociedade Brasileira de Diabetes (2019). O Diabetes Mellitus Tipo 2 corresponde a 90 a 95\% de todos os casos. Quanto a presença de agravos secundários, encontrou-se 70,4\% dos utentes com hipertensão arterial e 55,6\% com dislipidemia (Tabela 3). Sabe-se que utentes com Diabetes Mellitus Tipo 2 apresentam, em sua maioria, o fenótipo clínico de obesidade, hipertrigliceridemia, baixo colesterol da lipoproteína de alta densidade (HDL-c), hipertensão arterial, entre outros agravos (Gregg, Sattar \& Ali, 2016). 
Tabela 3 - Diagnóstico principal, secundário, complicações e pressão arterial nas consultas iniciais e finais.

Notas:

\begin{tabular}{|c|c|c|}
\hline PARÃMETROS & FA (n) & FR (\%) \\
\hline \multicolumn{3}{|c|}{ Diagnóstico principal } \\
\hline Diabetes mellitus Tipo 1 & 7 & $25,9 \%$ \\
\hline Diabetes mellitus Tipo 2 & 17 & $63,0 \%$ \\
\hline Informação ausente & 3 & $11,1 \%$ \\
\hline \multicolumn{3}{|c|}{ Diagnóstico secundário } \\
\hline Hipertensão arterial & 19 & $70,4 \%$ \\
\hline Dislipidemia & 15 & $55,6 \%$ \\
\hline Hipotireoidismo & 4 & $14,8 \%$ \\
\hline Doença e/ou evento cardiovascular & 5 & $18,5 \%$ \\
\hline Apresenta complicações & 8 & $29,6 \%$ \\
\hline Retinopatia diabética com perda parcial de visão & 3 & $11,1 \%$ \\
\hline Retinopatia diabética com perda total de visão & 1 & $3,7 \%$ \\
\hline Neuropatia diabética & 2 & $7,4 \%$ \\
\hline Nefropatia diabética com hemodiálise & 1 & $3,7 \%$ \\
\hline Nefropatia diabética sem hemodiálise & 1 & $3,7 \%$ \\
\hline Complicações macrovasculares ${ }^{1}$ & 1 & $3,7 \%$ \\
\hline Não apresenta complicações & 19 & $70,4 \%$ \\
\hline
\end{tabular}

\begin{tabular}{|c|c|c|}
\hline \multicolumn{3}{|c|}{ Medida casual da pressão arterial ${ }^{2}$} \\
\hline \multicolumn{3}{|c|}{ Consulta inicial $^{3}$} \\
\hline Hipotensão & 1 & $3,7 \%$ \\
\hline Normotensão & 17 & $63 \%$ \\
\hline Hipertensão & 9 & $33,3 \%$ \\
\hline \multicolumn{3}{|c|}{ Consulta final $^{3}$} \\
\hline Hipotensão & 1 & $3,7 \%$ \\
\hline Normotensão & 22 & $81,5 \%$ \\
\hline Hipertensão & 4 & $14,8 \%$ \\
\hline
\end{tabular}

${ }^{1}$ As complicações macrovasculares no utente com diabetes compreendem a doença arterial coronariana (DAC), o acidente vascular cerebral isquêmico (AVC) e a doença arterial periférica (DAP).

2 Classificação de acordo com Diretriz Brasileira de Hipertensão Arterial de 2018-2019.

${ }^{3}$ Valores de pressão arterial obtidos durante consultas mediante autorização do utente e presença de um farmacêutico habilitado.

Fonte: Perfil de prescrição e de utilização de medicamentos em utentes com diabetes mellitus atendidos via processos judiciais no Centro de Especialidades Médicas e Odontológicas (CEMO) do município de Belém, Pará.

29,6\% dos utentes apresentavam alguma complicação relacionada ao diabetes; dentre as quais, a prevalência de retinopatia diabética em 50\% (Tabela 3). Freitas (2016) encontrou 40,7\% dos utentes estudados com retinopatia diabética. Para utentes com este perfil, o CEMO disponibiliza caixas de medicamentos adaptadas com texturas diferentes para reconhecimento de seus medicamentos; sendo orientados a buscarem o apoio de um cuidador para assegurar o uso correto dos medicamentos.

70,4\% dos utentes relataram não ter complicação proveniente do Diabetes Mellitus (Tabela 3). Existem evidências de que indivíduos mau controlado ou não tratado adequadamente desenvolvem complicações. Ainda não está claro se as complicações do diabetes são resultantes da própria hiperglicemia ou de condições associadas; tais como, deficiência de insulina, excesso de glucagon, mudanças da osmolaridade, glicação de proteínas e alterações lipídicas ou da pressão arterial (SBD, 2019).

Importante registrar que os utentes foram monitorados quanto aos parâmetros fisiológicos; em especial, pressão arterial, glicemia capilar casual e peso (Tabela 3). Quanto a pressão arterial, observou-se o aumento de utentes com normotensão de $63 \%$ para $81,5 \%$ após as intervenções farmacêuticas. Para glicemia capilar casual observou-se uma redução de $215,29 \pm 80,56 \mathrm{mg} / \mathrm{dL}$ para $131 \pm 34,74 \mathrm{mg} / \mathrm{dL}$ (Tabela 3 ).

A Tabela 4 apresenta dados do monitoramento da glicemia capilar diariamente. O monitoramento do Diabetes Mellitus em qualquer faixa etária traz grandes benefícios por diminuir o risco de complicações agudas; tais como, cetoacidose 
e hipoglicemia e, possibilitar ajustes da insulina basal, seja no esquema de múltiplas doses de insulina, seja na bomba de infusão (SBD, 2019).

Tabela 4 - Valores de glicemia capilar casual no automonitoramento residencial, consulta inicial e final.

\begin{tabular}{|c|c|c|}
\hline PARÂMETROS & Média & $\mathbf{D P}^{\mathbf{1}}$ \\
\hline \multicolumn{3}{|c|}{ Glicemia capilar casual $^{2}$} \\
\hline Consulta inicial & $215,29 \mathrm{mg} / \mathrm{dL}$ & 80,56 \\
\hline Consulta final & $131 \mathrm{mg} / \mathrm{dL}$ & 34,74 \\
\hline \multicolumn{3}{|c|}{ Automonitoramento glicêmico após a consulta inicial } \\
\hline Jejum & 144,33 & 37,02 \\
\hline Pós prandial ${ }^{3}$ & 188,05 & 47,90 \\
\hline Hora de dormir ${ }^{4}$ & 180,12 & 53,86 \\
\hline Madrugada $^{5}$ & 148,75 & 72,73 \\
\hline \multicolumn{3}{|c|}{ Automonitoramento glicêmico após na consulta final } \\
\hline Jejum & 110,77 & 28,56 \\
\hline Pós prandial & 152,76 & 29,84 \\
\hline Hora de dormir & 161,33 & 34,19 \\
\hline Madrugada & 137,33 & 59,01 \\
\hline
\end{tabular}

Notas:

${ }^{1} \mathrm{DP}$ - Desvio Padrão.

${ }^{2}$ A glicemia plasmática casual é definida como aquela realizada a qualquer hora do dia, sem observar o intervalo da última refeição (SBD, 2019).

${ }^{3}$ Pós-prandial - Verificação 2 horas após a refeição.

${ }^{4}$ Aplicada apenas para utentes que a hora de dormir distanciava-se do horário pós-prandial noturno.

${ }^{5}$ Aplicada apenas para utentes que acordassem com mau estar.

Fonte: Perfil de prescrição e de utilização de medicamentos em utentes com diabetes mellitus atendidos via processos judiciais no Centro de Especialidades Médicas e Odontológicas (CEMO) do município de Belém, Pará.

Ao longo do estudo, quando solicitados pelos farmacêuticos, 62,9\% dos utentes apresentaram exames laboratoriais complementares atualizados. Dentre os exames, $88,3 \%$ estavam relacionados com a função renal, $88,9 \%$ com a função metabólica e 50\% ao parâmetro A1C (Tabela 5); dados imprescindíveis para o acompanhamento da evolução dos utentes em atendimento. Importante destacar que esses exames eram feitos em laboratórios particulares. Quanto às restrições alimentares (Tabela 5), encontrou-se restrição ao potássio, uma vez que o uso de diuréticos poupadores de potássio em utentes hipertensos e com redução de função renal pode acarretar hiperpotassemia. 
Tabela 5 - Exames laboratoriais complementares e restrições alimentares.

\begin{tabular}{|c|c|c|}
\hline PARÂMETROS & FA (n) & FR $(\%)$ \\
\hline \multicolumn{3}{|l|}{ Exames laboratoriais } \\
\hline Não apresentaram exames laboratoriais & 9 & $33,3 \%$ \\
\hline Apresentaram exames laboratoriais & 18 & $66,7 \%$ \\
\hline \multicolumn{3}{|l|}{ Exames complementares apresentados } \\
\hline Função renal & 15 & $88,3 \%$ \\
\hline Função hepatobiliar & 14 & $77,8 \%$ \\
\hline Função metabólica & 16 & $88,9 \%$ \\
\hline Glicose jejum & 17 & $94,4 \%$ \\
\hline Glicose pós-prandial & 9 & $50 \%$ \\
\hline Hemoglobina glicada & 13 & $72,2 \%$ \\
\hline Função muscular & 4 & $22,2 \%$ \\
\hline Função da tireoide & 2 & $11,1 \%$ \\
\hline Hemograma & 9 & $50 \%$ \\
\hline \multicolumn{3}{|l|}{ Restrições alimentares } \\
\hline Não apresentaram restrições & 11 & $40,7 \%$ \\
\hline Apresentaram restrições & 16 & 59,3 \\
\hline Potássio $^{1}$ & 10 & $62,5 \%$ \\
\hline Outros sais & 3 & $18,8 \%$ \\
\hline Lactose & 2 & $12,5 \%$ \\
\hline Água $^{2}$ & 1 & $6,2 \%$ \\
\hline
\end{tabular}

Notas: Indicadores Clínicos e Laboratoriais obtidos a partir dos próprios utentes.

${ }^{1}$ Restrição baseada em uso de fármacos que apresenta interação com potássio.

${ }^{2}$ Utente renal crônico em hemodiálise.

Fonte: Perfil de prescrição e de utilização de medicamentos em utentes com diabetes mellitus atendidos via processos judiciais no Centro de Especialidades Médicas e Odontológicas (CEMO) do município de Belém, Pará.

\section{Perfil de utilização de medicamentos}

Observou-se que para $100 \%$ dos utentes havia uma média de 6,29 ( $\pm 3,76)$ medicamentos prescritos. 91,2\% dos utentes apresentavam pelo menos um medicamento adjuvante; além das insulinas análogas prescritas. Desses 74,2\% apresentavam prescrições de hipoglicemiantes orais, $74,2 \%$ de anti-hipertensivos, $58 \%$ de hipolipemiantes e $41,9 \%$ de diuréticos (Tabela 6). 
Tabela 6 - Uso de insulinas análogas, classes terapêuticas adjuvantes, grau de conhecimento, adesão ao tratamento e perfil de uso de medicamentos.

\begin{tabular}{|c|c|c|}
\hline PARÂMETROS & FA (n) & FR (\%) \\
\hline \multicolumn{3}{|c|}{ Insulinas análogas em uso } \\
\hline Degludeca & 23 & $73,5 \%$ \\
\hline Glargina & 32 & $94 \%$ \\
\hline Detemir & 9 & $26,5 \%$ \\
\hline Asparte & 13 & $38 \%$ \\
\hline Lispro & 15 & $44,1 \%$ \\
\hline Glulisina & 14 & $41,2 \%$ \\
\hline Não faz uso de terapia adjuvante & 3 & $8,8 \%$ \\
\hline Faz uso de terapia adjuvante & 31 & $91,2 \%$ \\
\hline \multicolumn{3}{|c|}{ Classes terapêuticas de medicamentos adjuvantes em uso } \\
\hline Hipoglicemiantes orais & 23 & $74,2 \%$ \\
\hline Antihipertensivos & 23 & $74,2 \%$ \\
\hline Antidepressivos & 4 & $12,9 \%$ \\
\hline Anticoagulantes & 5 & $16,1 \%$ \\
\hline Diuréticos & 13 & $41,9 \%$ \\
\hline Hipolipemiantes & 18 & $58 \%$ \\
\hline Hormônio tireoidiano & 7 & $22,6 \%$ \\
\hline \multicolumn{3}{|c|}{ Grau de conhecimento sobre o tratamento } \\
\hline Excelente & 2 & $7,4 \%$ \\
\hline Bom & 16 & $59,3 \%$ \\
\hline Regular & 7 & $25,9 \%$ \\
\hline Péssimo & 2 & $7,4 \%$ \\
\hline \multicolumn{3}{|c|}{ Grau de adesão ao tratamento } \\
\hline Média Adesão & 4 & $14,8 \%$ \\
\hline Adesão & 23 & $85,2 \%$ \\
\hline Estatística & $\mathbf{N}^{1}$ & $\mathrm{Md}^{2} \pm \mathrm{DP}^{3}$ \\
\hline Média de medicamento prescrito por utente & 170 & $6,29 \pm 3,76$ \\
\hline Média de duplicidade terapêutica por utente & 7 & $0,26 \pm 0,452$ \\
\hline \multicolumn{3}{|c|}{ Média de potencial interação medicamento-medicamento por utente } \\
\hline Alta gravidade & 4 & $0,19 \pm 0,693$ \\
\hline Média gravidade & 147 & $12 \pm 28,68$ \\
\hline
\end{tabular}

\footnotetext{
Alta gravidade

Média de potencial interação medicamento-alimento por utente

Média gravidade

39

0

Notas:

${ }^{1}$ Número total de potenciais interações medicamento-medicamento e medicamento-alimento.

${ }^{2}$ Média

${ }^{3}$ Desvio Padrão.

Fonte: Perfil de prescrição e de utilização de medicamentos em utentes com diabetes mellitus atendidos via processos judiciais no Centro de Especialidades Médicas e Odontológicas (CEMO) do município de Belém, Pará.
}

A insulina glargina (Lantus®) foi encontrada em 94\% das prescrições; ou seja, a insulina de ação lenta mais prescrita (Tabela 6). Outrossim, estudos evidenciam que degludeca (Tresiba®) demonstra uma superioridade efetividade e uma menor incidência de eventos hipoglicêmicos em diferentes populações diabéticas quando comparada a glargina (Lantus®) (Marc et al., 2017; Zhang et al., 2018).

A insulina lispro (Humalog®) foi encontrada em 44,1\%, sendo a insulina de ação ultrarrápida mais prescrita. Liu et al., (2018) evidenciam que não há diferenças significantes nos valores de glicemia média de 24h, glicemia de jejum e incidência de hipoglicemia em utentes quando comparadas as insulinas lispro (Humalog®) e asparte (NovoRapid®) (Tabela $6)$.

Identificou-se 4 prescrições com potenciais interações medicamento-medicamento de alta gravidade e 147 potenciais interações medicamento-medicamento de média gravidade, sendo os utentes encaminhados para o profissional prescritor com 
sugestão de revisão de utilização de medicamentos e/ou de conciliação de medicamentos (Tabela 6). Não houve retorno dos prescritores.

Quanto às interações medicamento-alimento foram identificadas 39 potenciais interações (Tabela 6). Todos os utentes foram informados sobre os riscos e orientados a evitar tais alimentos. Identificou-se, também, 7 duplicidade terapêutica, para os quais também foram apresentadas proposta de revisão de utilização de medicamentos e/ou de conciliação de medicamentos (Tabela 6). Também, Não houve retorno dos prescritores. Prado, Francisco \& Barros (2016), encontraram em 299 idosos diabéticos - em uso de 2 ou mais medicamentos -, 413 potenciais interações medicamentosas, sendo que 53,1\% tiveram interações moderadas e 7,2\% interações graves.

59,3\% dos utentes demonstraram bom conhecimento do seu tratamento e $85,2 \%$ auto-referiram ter adesão ao tratamento (Tabela 6). Sabe-se que a adesão ao tratamento sofre influência de distintos fatores; entre eles, aspectos como renda familiar, nível de escolaridade, dificuldade de se lembrar dos horários, interrupção do tratamento, falta de conhecimento sobre os riscos da doença e o acesso aos medicamentos utilizados (Farias et al., 2019). Importante ressaltar que o cumprimento das metas clínicas está diretamente relacionado com a adesão ao tratamento.

\section{Dados e informações inerentes ao cuidado farmacêutico}

100\% dos utentes foram investigados sobre seu estado global de saúde, dos quais 85,2\% relataram queixas (Tabela 7). As principais queixas relatadas foram: insônia para 37,9\%, queixa sobre pés e/ou mãos inchadas para 31\% e dores de cabeça frequente para 27,6\%. Estes sinais e/ou sintomas podem estar relacionados a vários fatores; entre eles, o agravamento do diabetes, estresse excessivo e/ou reação a algum medicamento. Prado, Francisco e Barros (2016) destacam a importância da atenção aos relatos de queixas dos utentes.

Tabela 7 - Principais queixas de saúde autorrelatadas.

\begin{tabular}{|c|c|c|}
\hline PARÃMETROS & FA (n) & FR (\%) \\
\hline Não apresentaram queixas & 4 & $14,8 \%$ \\
\hline Apresentaram queixas & 23 & $85,2 \%$ \\
\hline \multicolumn{3}{|c|}{ Queixas apresentadas } \\
\hline Insônia & 10 & $37,0 \%$ \\
\hline Mãos e/ou pés inchados & 9 & $33,3 \%$ \\
\hline Dor de cabeça frequente & 8 & $29,6 \%$ \\
\hline Dores musculares & 6 & $22,2 \%$ \\
\hline Fadiga e/ou cansaço & 6 & $22,2 \%$ \\
\hline Problemas gastrointestinais & 3 & $11,1 \%$ \\
\hline Visão turva & 3 & $11,1 \%$ \\
\hline Ansiedade & 2 & $7,4 \%$ \\
\hline
\end{tabular}

Fonte: Perfil de prescrição e de utilização de medicamentos em utentes com diabetes mellitus atendidos via processos judiciais no Centro de Especialidades Médicas e Odontológicas (CEMO) do município de Belém, Pará.

Ao avaliar a farmacoterapia do utente, algumas questões eram postas, a fim de ter informações não apenas dos fármacos em uso, mas como eles foram prescritos, em que situações, se os usuários estavam satisfeitos com o tratamento ou não, entre outros fatores importantes para a avaliação e intervenção (Quadro 1). 
Quadro 1 - Conhecimento sobre o diagnóstico, prognóstico, farmacoterapia e satisfação do utente com o atendimento.

\begin{tabular}{|c|c|c|}
\hline PARÂMETROS & $\operatorname{SIM}(\%)$ & $\begin{array}{c}\mathbf{N} \tilde{A} \mathbf{O} \\
(\%)\end{array}$ \\
\hline O utente foi informado e sabe o diagnóstico da doença que está tratando? & $21(78 \%)$ & $6(22 \%)$ \\
\hline O utente foi esclarecido sobre o seu prognóstico? & $\begin{array}{c}20 \\
(74,1 \%)\end{array}$ & $\begin{array}{c}7 \\
(25,9 \%)\end{array}$ \\
\hline O utente foi informado e sabe para que serve $(\mathrm{m}) \mathrm{o}(\mathrm{s})$ medicamento(s) que irá utilizar e/ou utiliza? & $20(74 \%)$ & $7(26 \%)$ \\
\hline O utente foi informado e sabe quais os objetivos (resultados esperados) do tratamento estabelecido? & $20(74 \%)$ & $7(26 \%)$ \\
\hline O utente foi informado e sabe a(s) dose(s) que deve tomar do(s) medicamento(s)? & $\begin{array}{c}25 \\
(92,6 \%)\end{array}$ & $2(7,4 \%)$ \\
\hline O utente foi informado e sabe os horários de administração dos medicamentos? & $\begin{array}{c}26 \\
(96,3 \%) \\
\end{array}$ & $1(3,7 \%)$ \\
\hline O utente foi informado e sabe o modo correto de tomar os medicamentos? & $\begin{array}{c}23 \\
(85,2 \%)\end{array}$ & $\begin{array}{c}4 \\
(14,8 \%) \\
\end{array}$ \\
\hline O utente foi informado e sabe se irá ou não precisar que alguém lhe administre os medicamentos? & $\begin{array}{c}26 \\
(96,3 \%)\end{array}$ & $1(3,7 \%)$ \\
\hline $\begin{array}{l}\text { O utente foi informado e sabe se há qualquer restrição [alimentar, ingestão de bebidas alcoólicas, não } \\
\text { dirigir ou utilizar equipamentos que exigem total atenção e controle, entre outras] durante o uso dos } \\
\text { medicamentos? }\end{array}$ & $\begin{array}{c}13 \\
(48,2 \%)\end{array}$ & $\begin{array}{c}14 \\
(51,8 \%)\end{array}$ \\
\hline $\begin{array}{l}\text { O utente foi informado e sabe dizer se os medicamentos podem causar algum efeito colateral e o que } \\
\text { fazer caso isto ocorra? }\end{array}$ & $10(37 \%)$ & $17(63 \%)$ \\
\hline $\begin{array}{l}\text { O utente foi informado e sabe dizer se os medicamentos podem causar alguma reação adversa e/ou } \\
\text { evento adverso e o que fazer caso isto ocorra? }\end{array}$ & $\begin{array}{c}9 \\
(33,3 \%) \\
\end{array}$ & $\begin{array}{c}18 \\
(67,7 \%) \\
\end{array}$ \\
\hline $\begin{array}{l}\text { O utente foi informado e sabe o que deve fazer caso se esqueça de tomar o medicamento na hora } \\
\text { marcada? }\end{array}$ & $\begin{array}{c}16 \\
(59,2 \%)\end{array}$ & $\begin{array}{c}11 \\
(40,8 \%)\end{array}$ \\
\hline $\begin{array}{l}\text { O utente foi informado e sabe em que casos os medicamentos que usa podem interferir em exames } \\
\text { laboratoriais e o que fazer nestes casos? }\end{array}$ & $\begin{array}{c}5 \\
(18,5 \%)\end{array}$ & $\begin{array}{c}22 \\
(81,5 \%)\end{array}$ \\
\hline O utente se sente bem-informado sobre o tratamento que lhe foi prescrito? & $\begin{array}{c}23 \\
(85,2 \%) \\
\end{array}$ & $\begin{array}{c}4 \\
(14,8 \%) \\
\end{array}$ \\
\hline O utente acredita ser importante estar bem-informado sobre o seu tratamento? & $\begin{array}{c}24 \\
(88,9 \%)\end{array}$ & $\begin{array}{c}3 \\
(11,1 \%)\end{array}$ \\
\hline O utente foi esclarecido sobre a necessidade de mudanças de hábitos e/ou estilo de vida? & $\begin{array}{c}26 \\
(96,3 \%)\end{array}$ & $1(3,7 \%)$ \\
\hline O utente foi esclarecido sobre como será o seu tratamento farmacológico e não-farmacológico? & $\begin{array}{c}24 \\
(88,9 \%) \\
\end{array}$ & $\begin{array}{c}3 \\
(11,1 \%) \\
\end{array}$ \\
\hline O utente foi esclarecido sobre por quanto tempo usar e como usar os medicamentos prescritos? & $\begin{array}{c}22 \\
(81,5 \%)\end{array}$ & $\begin{array}{c}5 \\
(18,5 \%)\end{array}$ \\
\hline $\begin{array}{l}\text { O utente foi questionado se faz uso de outros medicamentos e/ou de outros tratamentos ao mesmo } \\
\text { tempo (concomitantes)? }\end{array}$ & $\begin{array}{c}20 \\
(74,1 \%)\end{array}$ & $\begin{array}{c}7 \\
(25,9 \%)\end{array}$ \\
\hline O utente ficou satisfeito com o atendimento médico e o tratamento estabelecido? & $\begin{array}{c}24 \\
(88,9 \%\end{array}$ & $\begin{array}{c}3 \\
(11,1 \%)\end{array}$ \\
\hline O utente ficou satisfeito com o atendimento farmacêutico e as informações e orientações prestadas? & $\begin{array}{c}27 \\
(100 \%) \\
\end{array}$ & $\begin{array}{c}0 \\
(0 \%)\end{array}$ \\
\hline O utente ficou satisfeito com o atendimento no CEMO? & $\begin{array}{c}27 \\
(100 \%)\end{array}$ & $\begin{array}{c}0 \\
(0 \%)\end{array}$ \\
\hline
\end{tabular}

Fonte: Perfil de prescrição e de utilização de medicamentos em utentes com diabetes mellitus atendidos via processos judiciais no Centro de Especialidades Médicas e Odontológicas (CEMO) do município de Belém, Pará.

Quanto ao conhecimento sobre o diagnóstico, prognóstico, farmacoterapia e satisfação do utente com o atendimento, $70,4 \%$ auto-referiram se sentir bem esclarecido sobre seu diagnóstico, $74,1 \%$ sobre o seu prognóstico, $96,3 \%$ sobre a necessidade de mudança de hábitos e/ou estilo de vida e 88,9\% sobre seu tratamento farmacológico e não farmacológico (Quadro 1). 74,1\% relataram terem sido questionados sobre seu tratamento; evitando, assim, prescrições com duplicidade terapêuticas e interações medicamentosas. Importante destacar que quanto melhor informado estiver o utente, melhor a eficiência do tratamento. A terapêutica alinhada com as necessidades individuais proporcionam a compreensão da doença e melhoram a qualidade de vida (Almeida \& Almeida, 2018). 
No Quadro 1 há evidencia de que os utentes estão bem-informados sobre sua estratégia farmacoterapêutica, onde 59,3\% apresentaram o nível bom na classificação do grau de conhecimento. Batista \& Braga (2017), relataram que 100\% dos utentes haviam sido informados e sabiam a indicação dos medicamentos que utilizam e, $80 \%$ haviam sido informados e sabiam sobre os objetivos e resultados esperados do tratamento.

Quanto ao Percentual de Medicamentos Adequados, 201 medicamentos prescritos apresentaram um maior percentual de adequação quanto aos critérios avaliados (Quadro 2). Para o critério "Interação Medicamento-Medicamento" 33,8\% dos medicamentos foram categorizados como fracamente apropriados; uma vez que os riscos desse tipo de interação é alto. Para o critério "Eficiência" que avalia o custo do medicamento em comparação com outros agentes de igual eficácia e segurança foi achado que $37,8 \%$ eram fracamente apropriados.

Quadro 2 - Percentual de Medicamentos Adequados.

\begin{tabular}{|l|c|c|c|}
\hline \multicolumn{1}{|c|}{ PARÂMETROS } & I* & PA** & $\mathbf{A}^{* * *}$ \\
\hline Indicação: sinal, sintoma, doença ou condição para a qual o medicamento é prescrito. & 0 & $18(9 \%)$ & $\begin{array}{c}183 \\
(91 \%)\end{array}$ \\
\hline Eficácia e efetividade: potencial em produzir um resultado benéfico. & 0 & $18(9 \%)$ & $\begin{array}{c}183 \\
(91 \%)\end{array}$ \\
\hline Dosagem: quantidade total do medicamento tomado por período de 24 horas. & 0 & $15(7,4 \%)$ & $\begin{array}{c}186 \\
(92,6 \%)\end{array}$ \\
\hline Instruçães: instruç̃̃es ao utente para o uso adequado do medicamento. & 0 & $8(3,9)$ & $\begin{array}{c}193 \\
(96 \%)\end{array}$ \\
\hline Praticidade e adesão ao tratamento: capacidade de ser usada ou colocada em prática. & 0 & $19(9,5 \%)$ & $\begin{array}{c}182 \\
(90,5 \%)\end{array}$ \\
\hline $\begin{array}{l}\text { Interação medicamento-medicamento: o efeito que a administração de um medicamento } \\
\text { tem em outro medicamento; interações prejudiciais. }\end{array}$ & 0 & $\begin{array}{c}68 \\
(33,8 \%)\end{array}$ & $\begin{array}{c}133 \\
(61,2 \%)\end{array}$ \\
\hline $\begin{array}{l}\text { Interação doença-doença: o efeito que um medicamento tem em uma doença ou condição } \\
\text { pré-existente; um significado clínico como uma interação prejudicial. }\end{array}$ & 0 & $\begin{array}{c}39 \\
(19,4 \%)\end{array}$ & $\begin{array}{c}162 \\
(80,6 \%)\end{array}$ \\
\hline $\begin{array}{l}\text { Duplicidade desnecessária: prescrição não benéfica ou arriscada de dois ou mais } \\
\text { medicamentos da mesma classe química ou farmacológica. }\end{array}$ & 0 & $\begin{array}{c}29 \\
(14,4 \%)\end{array}$ & $\begin{array}{c}172 \\
(85,6 \%)\end{array}$ \\
\hline $\begin{array}{l}\text { Duração: duração da terapia. } \\
\text { Eficiência (custo): custo do medicamento em comparação com outros agentes de igual } \\
\text { eficácia e segurança. }\end{array}$ & 0 & $\begin{array}{c}29 \\
(14,4 \%)\end{array}$ & $\begin{array}{c}172 \\
(85,6 \%)\end{array}$ \\
\hline
\end{tabular}

Nota: $\mathrm{I}=$ Inadequado*; PA = Pouco adequado**; A = Apropriado***.

Fonte: Perfil de prescrição e de utilização de medicamentos em utentes com diabetes mellitus atendidos via processos judiciais no Centro de Especialidades Médicas e Odontológicas (CEMO) do município de Belém, Pará.

Quanto aos Resultados Negativos Associados aos Medicamentos (RNM) (Quadro 3), observou-se que 67,6\% estão relacionados a problemas de saúde não tratados, $20,6 \%$ a duplicidade de medicamentos, $17,7 \%$ a interações medicamentomedicamento e $14,7 \%$ a probabilidade de efeitos adversos aos medicamentos. 
Quadro 3 - Resultados Negativos Associados ao Medicamento.

\begin{tabular}{|c|c|}
\hline PARÂMETROS & $\mathbf{N}(\%)$ \\
\hline \multicolumn{2}{|l|}{ Classificação do RNM } \\
\hline$\%$ de problema de saúde não tratado & $23(67,6 \%)$ \\
\hline$\%$ de efeito de medicamento desnecessário & $3(8,8 \%)$ \\
\hline \% de inefetividade não quantitativa & 0 \\
\hline$\%$ de inefetividade quantitativa & 0 \\
\hline$\%$ de insegurança não quantitativa & 0 \\
\hline$\%$ de insegurança quantitativa & 0 \\
\hline \multicolumn{2}{|l|}{ Medicamentos implicados nos RNM e os seus respectivos percentuais } \\
\hline$\%$ relacionado a dose & $4(11,8 \%)$ \\
\hline \% relacionado a via de administração & 0 \\
\hline \% relacionado a frequência de administração & 0 \\
\hline$\%$ relacionado a tempo de tratamento & $6(17,7 \%)$ \\
\hline \multicolumn{2}{|l|}{ Causas dos Problemas Relacionados aos Medicamentos (PRMs) } \\
\hline \% de erro na administração do medicamento & 0 \\
\hline$\%$ de características pessoais & 0 \\
\hline$\%$ de conservação inadequada & 0 \\
\hline \% de contraindicação & 0 \\
\hline \% de dose, via de administração, frequência de administração e tempo de tratamento inadequados & $6(17,7 \%)$ \\
\hline$\%$ de duplicidade & $7(20,6 \%)$ \\
\hline \% de erro de dispensação & 0 \\
\hline$\%$ de erro de prescrição & 0 \\
\hline \% de não cumprimento do tratamento (não adesão) & 0 \\
\hline \% de interações de medicamentos-medicamentos & $6(17,7 \%)$ \\
\hline$\%$ de outros problemas de saúde que afetam o tratamento & 0 \\
\hline$\%$ de probabilidade de efeitos adversos & $5(14,7 \%)$ \\
\hline$\%$ de outras causas & 0 \\
\hline
\end{tabular}

Fonte: Perfil de prescrição e de utilização de medicamentos em utentes com diabetes mellitus atendidos via processos judiciais no Centro de Especialidades Médicas e Odontológicas (CEMO) do município de Belém, Pará.

Quanto à necessidade, efetividade e segurança dos medicamentos (Quadro 4), observou-se que 67,6\% de problemas de saúde não tratado, a exemplo de doenças secundárias, estava relacionado ao utente não ter acesso aos medicamentos necessários para o tratamento dos agravos secundários. 
Quadro 4 - Necessidade, efetividade e segurança dos medicamentos.

\begin{tabular}{|c|c|}
\hline PARÂMETROS & $\mathbf{N}(\%)$ \\
\hline \multicolumn{2}{|l|}{ NECESSIDADE } \\
\hline $\begin{array}{l}\text { \% de problema de saúde não tratado: o utente sofre um problema de saúde associado ao não recebimento de } \\
\text { medicamento }\end{array}$ & $\begin{array}{c}23 \\
(67,6 \%)\end{array}$ \\
\hline $\begin{array}{l}\text { \% de efeito de um medicamento não necessário: o utente sofre um problema de saúde ao receber um } \\
\text { medicamento que não necessita }\end{array}$ & 0 \\
\hline \multicolumn{2}{|l|}{ EFETIVIDADE } \\
\hline $\begin{array}{l}\text { \% de inefetividade não qualitativa: o utente sofre um problema de saúde associado a uma inefetividade não } \\
\text { qualitativa do medicamento }\end{array}$ & 0 \\
\hline $\begin{array}{l}\text { \% de inefetividade quantitativa: o utente sofre um problema de saúde associado a uma inefetividade } \\
\text { quantitativa do medicamento }\end{array}$ & 0 \\
\hline \multicolumn{2}{|l|}{ SEGURANÇA } \\
\hline $\begin{array}{l}\text { \% de segurança não quantitativa: o utente sofre um problema de saúde associado a uma insegurança não } \\
\text { quantitativa do medicamento }\end{array}$ & 0 \\
\hline $\begin{array}{l}\text { \% de segurança quantitativa: o utente sofre um problema de saúde associado a uma insegurança quantitativa do } \\
\text { medicamento }\end{array}$ & 0 \\
\hline
\end{tabular}

Fonte: Perfil de prescrição e de utilização de medicamentos em utentes com diabetes mellitus atendidos via processos judiciais no Centro de Especialidades Médicas e Odontológicas (CEMO) do município de Belém, Pará.

$\mathrm{R} \$ 454,87$ foi o custo médio mensal dos tratamentos dos utentes (Quadro 5). Não houve como comparar os valores antes e após as propostas de revisão de utilização de medicamentos e/ou conciliação de medicamentos; visto que, não houve retorno dos prescritores. Um fato relevante quando se trata de equipes multidisciplinares.

Quadro 5 - Custo médio mensal dos tratamentos dos utentes.

\begin{tabular}{|l|c|c|}
\hline \multicolumn{1}{|c|}{ PARÂMETROS } & Média (R\$) & $\mathrm{DP}^{\mathbf{1}}(\mathrm{R} \$)$ \\
\hline Custo médio mensal & 454,87 & 164,30 \\
\hline
\end{tabular}

Nota: ${ }^{1}$ Com base nos valores do Banco de Preços do Ministério da Saúde atualizados no dia 22 de maio de 2019.

Fonte: Perfil de prescrição e de utilização de medicamentos em utentes com diabetes mellitus atendidos via processos judiciais no Centro de Especialidades Médicas e Odontológicas (CEMO) do município de Belém, Pará - Pesquisa de campo.

Os utentes foram classificados como tendo boa qualidade de vida (Quadro 6); atingindo uma média acima de 3. Entretanto, quando se avalia individualmente cada questão, aparecem aspectos em que o utente precisaria de maior atenção. Destaca-se no Domínio 1 (Físico) a dor física e o aspecto financeiro relacionados ao bem-estar. No Domínio 2 (Psicológico) atenta-se para a presença de sentimentos negativos, o qual pode estar relacionada a presença de depressão e ansiedade; mesmo que não oficialmente diagnosticadas. 
Quadro 6 - Qualidade de vida autorreferido (WHOQOL).

\begin{tabular}{|c|c|c|}
\hline PARÂMETROS & $\mathbf{M}^{1}$ & $\mathbf{D P}^{2}$ \\
\hline \multicolumn{3}{|l|}{ Questões Gerais } \\
\hline Como você avaliaria sua qualidade de vida? & 3,88 & 0,85 \\
\hline Quão satisfeito você está com a sua saúde? & 3,00 & 1,07 \\
\hline Valores Gerais do Domínio & 3,44 & 0,63 \\
\hline \multicolumn{3}{|l|}{ Domínio 1 - Domínio Físico } \\
\hline 1 - Em que medida você acha que sua dor (física) impede você de fazer o que você precisa? & 2,55 & 1,15 \\
\hline 2 - O quanto você precisa de algum tratamento médico para levar sua vida diária? & 3,33 & 1,24 \\
\hline 3 - O quanto você aproveita a vida? & 3,66 & 0,83 \\
\hline 9 - Você é capaz de aceitar sua aparência física? & 3,85 & 1,13 \\
\hline 10 - Você tem dinheiro suficiente para satisfazer suas necessidades? & 2,37 & 0,79 \\
\hline 11- Quão disponíveis para você estão às informações que precisa no seu dia a dia? & 3,40 & 1,01 \\
\hline 12 - Em que medida você tem oportunidades de atividades de lazer? & 3,00 & 0,78 \\
\hline Valores Gerais do Domínio & 3,13 & 0,55 \\
\hline \multicolumn{3}{|l|}{ Domínio 2 - Domínio Psicológico } \\
\hline 4 - Em que medida você acha que sua vida tem sentido? & 4,07 & 0,96 \\
\hline 5 - O quanto você consegue se concentrar? & 3,55 & 1,01 \\
\hline 6 - Quão seguro (a) você se sente em sua vida? & 3,59 & 0,97 \\
\hline 7 - Quão saudável é o seu ambiente físico? & 3,25 & 0,94 \\
\hline 8- Você tem energia suficiente para seu dia a dia? & 3,55 & 0,93 \\
\hline 24 - Com que frequência você tem sentimentos negativos & 2,14 & 1,17 \\
\hline Valores Gerais do Domínio & 3,36 & 0,65 \\
\hline \multicolumn{3}{|l|}{ Domínio 3 - Relações Sociais } \\
\hline 13 - Quão bem você é capaz de se locomover? & 3,88 & 0,85 \\
\hline 14 - Quão satisfeito você está com seu sono? & 3,00 & 1,07 \\
\hline 15 - Quão satisfeito você está com sua capacidade de desempenhar atividades do seu dia a dia? & 3,44 & 1,09 \\
\hline Valores Gerais do Domínio & 3,44 & 0,44 \\
\hline \multicolumn{3}{|l|}{ Domínio 4 - Meio Ambiente } \\
\hline 16 - Quão satisfeito você está com sua capacidade de trabalho? & 3,55 & 1,12 \\
\hline 17 - Quão satisfeito você está consigo mesmo? & 3,88 & 0,97 \\
\hline 18 - Quão satisfeito você está com suas relações pessoais? & 3,96 & 0,76 \\
\hline 19 - Quão satisfeito você está com sua vida sexual? & 3,29 & 1,03 \\
\hline 20 - Quão satisfeito você está com o apoio que você recebe de seus amigos? & 3,55 & 0,97 \\
\hline 21 - Quão satisfeito você está com as condições do local onde mora? & 3,51 & 1,16 \\
\hline 22 - Quão satisfeito você está com o seu acesso aos serviços de saúde? & 3,66 & 1,1 \\
\hline 23 - Quão satisfeito você está como seu meio de transporte? & 2,92 & 1,07 \\
\hline Valores Gerais do Domínio & 3,63 & 0,33 \\
\hline
\end{tabular}

Nota: ${ }^{1}$ Média; ${ }^{2}$ Desvio Padrão.

Observação: Média individual ou global acima de 3,00 é considerado boa qualidade de vida.

Fonte: Perfil de prescrição e de utilização de medicamentos em utentes com diabetes mellitus atendidos via processos judiciais no Centro de Especialidades Médicas e Odontológicas (CEMO) do município de Belém, Pará.

Correa et al. (2017) registram que distintas variáveis influenciam na qualidade de vida dos diabéticos; com exceção, do tempo de diagnóstico e sexo. A idade influencia no valor de hemoglobina glicada, adesão à dieta alimentar e a prática de atividade física, evidenciando que a educação em saúde é um componente fundamental para o tratamento do Diabetes Mellitus.

Por fim, os achados aqui apresentados, oportuniza aos gestores e profissionais reflexionarem sobre os cuidados aos utentes com Diabetes Mellitus, de forma a qualificar os serviços, aprimorando as linhas de cuidados; em especial, a do cuidado farmacêutico. 


\section{Dificuldades, limitações e viés}

$\checkmark$ Dificuldade quanto a disponibilidade de dados e informações laboratoriais atualizadas.

$\checkmark$ Dificuldade quanto as relações interprofissionais no contexto da prática clínica; em especial, quanto ao retorno às propostas de intervenções farmacêuticas devido à ausência de médico endocrinologista.

$\checkmark$ Limitações de espaço físico apropriado para realização das consultas farmacêuticas; dificultando o acolhimento e o atendimento dos utentes.

$\checkmark$ Dificuldade e/ou limitação física e/ou financeira para a mobilidade urbana dos utentes para a acessibilidade ao CEMO.

$\checkmark$ Devido ao desenho do estudo - amostragem por conveniência - não foi possível análises estatísticas para testar associações entre a variável dependente e as variáveis independentes; assim, como quais as variáveis independentes influenciam a qualidade de vida dos utentes.

\section{Considerações Finais}

Infere-se que as intervenções farmacêuticas demonstraram ser efetivas quanto ao cuidado farmacêutico e a qualidade de vida do utente. Nesta direção, propõe-se reflexionar sobre a importância não só da acessibilidade as insulinas análogas via judicial; mas, imprescindivelmente da qualidade do acesso aos serviços de cuidados ofertados. Sugere-se aprimorar os estudos em outras faixas etárias.

\section{Conflitos de interesse}

Os autores registram não haver conflito de interesse.

\section{Agradecimentos}

Às farmacêuticas do Centro de Especialidades Médico Odontológica da Secretaria Municipal de Saúde (SESMA) da Prefeitura Municipal de Belém (PMB): Edivana Maciel dos Santos Barral, Paula Doriani dos Santos Bormann e Telma Lúcia Araújo Ferreira e aos gestores, técnicos-administrativos e utentes que participaram do estudo.

\section{Nota}

Estudo complementar sobre cuidado farmacêutico com "72 utentes acima de 60 anos de idade com Diabetes Mellitus (Tipo 1 e Tipo 2) em uso de uma ou mais insulinas análogas - lispro (Humalog®), asparte (NovoRapid®), glulisina (Lispro®), glargina (Lantus®), detemir (Levemir®), degludeca (Tresiba®) - atendidos sob demanda judicial no CEMO”, que estava em realização no período de 05 de agosto de 2019 a 31 de julho de 2020, foi descontinuado em função do Sars-CoV-2, causador da atual Pandemia de Covid-19.

\section{Referências}

Almeida, J. S. \& Almeida, J. M. (2018). A educação em saúde e o tratamento do diabetes mellitus tipo 2 em uma unidade de família. Revista da Faculdade de Ciências Médicas de Sorocaba. 20(1), 13-17. 10.23925/1984-4840.2018v20i1a4

Arruda, S. C. (2017). Análise sobre a judicialização da saúde no Estado de Mato Grosso no período de 2011-2012. Caderno Ibero-Americano de Direito Sanitário. 6(1), 86-111. http://dx.doi.org/10.17566/ciads.v6i1.308

Batista, A. V. A. \& Braga, P. H. C. (2017). Perfil de utilização de medicamentos em discentes que utilizam o Serviço de Assistência Psicossocial da Universidade Federal do Pará. Belém. Graduação (Trabalho de Conclusão de Curso). Faculdade de Farmácia. Instituto de Ciências da Saúde. Universidade Federal do Pará. Pará. 2017. 75p. 
Belém. (2015). Conselho Municipal de Saúde. Protocolo clínico para atendimento de análogos de insulina e antidiabéticos orais/injetáveis para utentes portadores de diabetes mellitus da rede pública de saúde do município de Belém, Pará. 21p.

Brandão, Y. H. C., Bormann, P. D. S., Queiroz, A. N. \& Soler, O. (2021). Cumprimento do protocolo clínico para atendimento de utentes de análogos de insulina em portadores de Diabetes Mellitus via judicial em Belém, Pará. Research, Society and Development. 10(1), e31310111800, ISSN 2525-3409. DOI: http://dx.doi.org/10.33448/rsd-v10i1.117800.

Brasil. (2012). Conselho Nacional de Saúde. Resolução n 466 de 12 de dezembro de 2012. Aprova as diretrizes e normas regulamentadoras de pesquisas envolvendo seres humanos. Brasília. Distrito Federal.

Brasil. (2014). Insulinas análogas de longa ação: diabetes melitus tipo II. Brasília: Ministério da Saúde. Relatório de Recomendação da Comissão Nacional de Incorporação de Tecnologias no SUS (CONITEC $\left.-\mathrm{n}^{\circ} 103\right)$. Brasília. DF.

Brasil. (2014). Insulinas análogas para diabetes mellitus tipo I. Brasília; Ministério da Saúde: Relatório de Recomendação da Comissão Nacional de Incorporação de Tecnologias no SUS (CONITEC - nº 114). Brasília. DF.

Brasil. (2014). Ministério da Saúde. Secretaria de Ciência, Tecnologia e Insumos Estratégicos. Departamento de Assistência Farmacêutica e Insumos Estratégicos. Serviços farmacêuticos na atenção básica à saúde. Brasília, 108p. il. - (Cuidado farmacêutico na atenção básica ; caderno 1). ISBN 978-85-3342196-7

Brasil. (2016). Ministério da Saúde. Secretaria de Ciência, Tecnologia e Insumos Estratégicos, Departamento de Ciência e Tecnologia. Síntese de evidências para políticas de saúde: adesão ao tratamento medicamentoso por utentes portadores de doenças crônicas. Brasília: Ministério da Saúde, 52p.

Brasil. (2016). Insulinas análogas de ação rápida para diabetes mellitus tipo I. Brasília; Ministério da Saúde: Relatório de Recomendação da Comissão Nacional de Incorporação de Tecnologias no SUS (CONITEC). Brasília. DF.

Brasil. (2016). Conselho Nacional de Saúde. Resolução n ${ }^{\circ}$ 510, de 07 de abril de 2016. Dispõe sobre as normas aplicáveis a pesquisas em Ciências Humanas e Sociais cujos procedimentos metodológicos envolvam a utilização de dados diretamente obtidos com os participantes ou de informações identificáveis ou que possam acarretar riscos maiores o que os existentes na vida cotidiana. Brasília. Distrito Federal.

Brasil. (2019). Insulinas análogas de ação prolongada para o tratamento de diabetes mellitus tipo II. Brasília; Ministério da Saúde: Relatório de Recomendação da Comissão Nacional de Incorporação de Tecnologias no SUS (CONITEC - nº 434). Brasília. DF.

Brasil. (2019). Ministério da Saúde. Secretaria de Ciência, Tecnologia e Insumos Estratégicos, Departamento de Ciência e Tecnologia. Portaria no 11, de 25 de fevereiro de 2019. Torna pública a decisão de não incorporar as insulinas análogas de ação prolongada para o tratamento de diabetes mellitus tipo II, no âmbito do Sistema Único de Saúde - SUS. Brasília. DF.

Brasil. (2020). Ministério da Saúde. Secretaria de Atenção Primária à Saúde. Departamento de Promoção da Saúde. Cuidado Farmacêutico na Atenção Básica: aplicação do método clínico - Brasília: Ministério da Saúde. Conteúdo: v. 1. O Cuidado farmacêutico no contexto do sistema de saúde. v. 2. Competências dos farmacêuticos para o Cuidado Farmacêutico. v. 3. Método clínico: acolhimento e coleta de dados. v. 4. Método clínico: avaliação e identificação dos problemas relacionados à farmacoterapia. v. 5. Método clínico: plano de cuidado, monitoramento e avaliação das metas estabelecidas.

Caires, E. S. (2016). Religião e espiritualidade de utentes internados na clínica médica do Hospital Renato Azeredo em Nanuque, Minas Gerais. Programa de Mestrado Profissional em Ciências da Religião. Faculdade Unida de Vitória. Dissertação (mestrado profissional). 117 p.

Conselho Federal de Farmácia (CFF). (2016). Programa de Suporte ao Cuidado Farmacêutico na Atenção à Saúde - PROFAR / Conselho Federal de Farmácia. - Brasília: Conselho Federal de Farmácia. 76 p. il. ISBN: 978-85-89924-18-4.

Corrêa, K., Gouvêa, G. R., Silva, M. A. V., Possobon, R. F., Barbosa, L. F. L. N., Pereira, A. C., Miranda, L. G. \& Cortellazzi, K. L. (2017). Qualidade de vida e características dos utentes diabéticos. Ciência \& Saúde Coletiva. 22(3), 921-930. https://doi.org/10.1590/1413-81232017223.24452015

Farias, E. B. O., Espinheira, M. J. C. L., Santana, N. R. S. \& Rodrigues, R. L. A. (2019). Adesão terapêutica em utentes diabéticos em uso de medicamentos. Id on Line Rev. Mult. Psic. 13(43), 407-415. ISSN 1981-1179

Fleck, M. P. A., Louzada, S., Xavier, M., Chachamovich, E., Vieira, G., Santos, L. \& Pinzon, V. (2000). Aplicação da versão em português do instrumento abreviado de avaliação da qualidade de vida "WHOQOL-bref". Rev. Saúde Pública. 34(2), 178-183. https://doi.org/10.1590/S0034-89102000000200012

Freitas, P. S. (2016). Perfil de utentes com Diabetes Mellitus tipo 1 em tratamento com insulinas convencionais e avaliação da qualidade de vida. Programa de Pós-Graduação em Ciências Farmacêuticas (Dissertação). Setor de Ciências Biológicas e da Saúde. Departamento de Ciências Farmacêuticas. Universidade Estadual de Ponta Grossa. Ponta Grossa, Mato Grosso do Sul.

Freitas, W. R. S. \& Jabbour, C. J. C. (2011). Utilizando estudo de caso(s) como estratégia de pesquisa qualitativa: boas práticas e sugestões. Estudo \& Debate. 18(2), 07-22. ISSN 1983-036X.

Fullerton, B., Siebenhofer, A., Jeitler, K., Horvath, K., Semlitsch, T., Berghold, A., Plank, J., Pieber, T. R. \& Gerlach, F. M. (2016). Short-acting insulin analogues versus regular human insulin for adults with type 1 diabetes mellitus. Cochrane Database Syst Ver. 6:CD012161.

Gregg, E. W., Sattar N. \& Ali, M. K. (2016). The changing face of diabetes complications. Lancet Diabetes Endocrinol. 4(6), 537-547. DOI: https://doi.org/10.1016/S2213-8587(16)30010-9

Liu, B. L., Yin, G. P., Li, F. F., Hu, Y., Wu, J., Chen, M., Ye, L., Su, X. \& Ma, J. (2018). Comparison of Efficacy and Safety of Lispro and Aspart Evaluated by Continuous Glucose Monitoring System in Patients with Newly Diagnosed Type 2 Diabetes. International Journal of Endocrinology. 2018:2087960. DOI: 10.1155/2018/2087960. 
Research, Society and Development, v. 10, n. 4, e49510414338, 2021

(CC BY 4.0) | ISSN 2525-3409 | DOI: http://dx.doi.org/10.33448/rsd-v10i4.14338

Marc, E., Billings, L. K., Håkan-Bloch, J., Slothuus, U., Abrahamsen, T. J., Andersen, A. \& Jansen, J. P. (2018) Uma comparação indireta do tratamento da eficácia da insulina degludec / liraglutida (IDegLira) e da insulina glargina / lixisenatida (iGlarLixi) em utentes com diabetes tipo 2 não controlada com insulina basal. Journal of Medical Economics. 21(4), 340-347. DOI: 10.1080 / 13696998.2017.1409228

Masmiquel, L., Leiter, L. A., Vidal, J., Bain, S., Petrie, J., Franek, E., Raz, I., Comlekci, A., Jacob, S., van Gaal, L., Baeres, F. M. M.; Marso, S. P. \& Eriksson, M. (2016). Leader 5: prevalence and cardiometabolic impact of obesity in cardiovascular high-risk patients with type 2 diabetes mellitus: baseline global data from the Leader Trial. Cardiovasc Diabetol. 10,15-29. 10.1186/s12933-016-0341-5

Minayo, M. C. S. (2012). Análise qualitativa: teoria, passos e fidedignidade. Ciênc. Saúde Coletiva. 17(3), 621-626. https://doi.org/10.1590/S141381232012000300007.

O'Neill, J. et al. (2013). Applying an equity lens to interventions: using PROGRESS ensures consideration of socially stratifying factors to illuminate inequities in health. Journal of Clinical Epidemiology. 67(1), 56 - 64. https://doi.org/10.1016/j.jclinepi.2013.08.005

Pará. (2016). Poder Judiciário. Tribunal Regional Federal da Primeira Região Seção Judiciária do Estado do Pará. Processo N 0006454-87.2008.4.01.3900, de 05 de abril de 2016. Trata de ação civil pública com pedido de tutela antecipada para que o município forneça as insulinas análogas e de todos os insumos necessários à sua aplicação (seringas com agulha acoplada para aplicação de insulina, tiras reagentes de medida de glicemia capilar e lancetes para punção digital), desde que que comprovem a real necessidade do uso desses medicamentos. Belém, Pará.

Patel, D. K., Laloo, D. \& Hemalatha, S. (2012). Natural medicines from plant source used for therapy of diabetes mellitus: An overview of its pharmacological aspects. Asian Pacific Journal of Tropical Disease. 2(3), 239-250. https://doi.org/10.1016/S2222-1808(12)60054-1

Prado, M. A. M. B., Francisco, P. M. S. B. \& Barros, M. B A. (2016). Diabetes em idosos: uso de medicamentos e risco de interação medicamentosa. Ciênc. saúde coletiva. 21(11), 3447-3458. https://doi.org/10.1590/1413-812320152111.24462015

Ramos, R. S., Gomes, A. M. T., Guimarães, R. M. \& Santos, E. I.. (2017). A judicialização da saúde contextualizada na dimensão prática das representações sociais dos profissionais de saúde. Revista de Direito Sanitário. 12(2), 18-38. http://dx.doi.org/10.11606/issn.2316-9044.v18i2p18-38.

Sociedade Brasileira de Diabetes (SBD). (2019). Diretrizes 2019-2020. Forti, A. C., et al. (Org.). Diretrizes da Sociedade Brasileira de Diabetes 2019-2020. Copyright ( 2019. Sociedade Brasileira de Diabetes. Clannad Ed. Cient. 489p.

Soler, O., Sinimbu, A. V., Figueredo, D. C. de, Vieira, H. K. dos S., Galucio, N. C. da R., Pinheiro, P. de N. Q., Sena, S. S. I., Silva, V. G.; Vieira, V. M. B., \& Ledo, Y. (2018). A reorientação da assistência farmacêutica na secretaria municipal de saúde de Belém (PA): Relato de experiência. Revista Eletrônica de Farmácia. 14(4), 41-55. ISSN 1808-0804. https://doi.org/10.5216/ref.v14i4.45427

Thiollent, M. (2011). Metodologia da pesquisa-Ação. (18a ed.), Cortez.

Vieira, L. G. (2017). O uso de fitoterápicos e plantas medicinais por utentes diabéticos. Brasília. Graduação (Trabalho de Conclusão de Curso). Amato, A. A. (Orientadora). Universidade de Brasília. Saúde. Brasília. Distrito Federal.

Yin, R. K. (2015). Estudo de caso: planejamento e métodos. (5a ed.), Bookman. 320p.

Zhang, X., Zhang, X., Xu, B. et al. (2018). Comparative safety and efficacy of insulin degludec with insulin glargine in type 2 and type 1 diabetes: a metaanalysis of randomized controlled trials. Acta Diabetol. 55, 429-441. https://doi.org/10.1007/s00592-018-1107-1 